\title{
A dried blood spot mass spectrometry metabolomic approach for rapid breast cancer detection
}

\author{
This article was published in the following Dove Press journal: \\ OncoTargets and Therapy \\ II March 2016 \\ Number of times this article has been viewed
}

\author{
Qingjun Wang ${ }^{1,2, *}$ \\ Tao Sun ${ }^{3, *}$ \\ Yunfeng Cao ${ }^{1,2,4,5}$ \\ Peng Gao $2,4,6$ \\ Jun Dong ${ }^{2,4}$ \\ Yanhua Fang ${ }^{2}$ \\ Zhongze Fang² \\ Xiaoyu Sun ${ }^{2}$ \\ Zhitu Zhu', \\ 'Oncology Department 2, The \\ First Affiliated Hospital of Liaoning \\ Medical University, ${ }^{2}$ Personalized \\ Treatment and Diagnosis Research \\ Center, The First Affiliated Hospital \\ of Liaoning Medical University and \\ Dalian Institute of Chemical Physics, \\ Chinese Academy of Sciences, \\ Jinzhou, ${ }^{3}$ Department of Internal \\ Medicine I, Cancer Hospital of China \\ Medical University, Liaoning Cancer \\ Hospital \& Insititute, Shenyang, ${ }^{4} \mathrm{CAS}$ \\ Key Laboratory of Separation Science \\ for Analytical Chemistry, Dalian \\ Institute of Chemical Physics, Chinese \\ Academy of Sciences, Dalian, ${ }^{5}$ Key \\ Laboratory of Contraceptives and \\ Devices Research (NPFPC), Shanghai \\ Engineer and Technology Research \\ Center of Reproductive Health \\ Drug and Devices, Shanghai Institute \\ of Planned Parenthood Research, \\ Shanghai, ${ }^{6} \mathrm{Clinical}$ Laboratory, Dalian \\ Sixth People's Hospital, Dalian, \\ People's Republic of China \\ *These authors contributed equally \\ to this work
}

Correspondence: Zhitu Zhu The First Affiliated Hospital of Liaoning Medical University, Jinzhou 121000 , People's Republic of China Fax +86 4164605049 Email kez_dicp@163.com

\begin{abstract}
Objective: Breast cancer (BC) is still a lethal threat to women worldwide. An accurate screening and diagnosis strategy performed in an easy-to-operate manner is highly warranted in clinical perspective. Besides the routinely focused protein markers, blood is full of small molecular metabolites with diverse structures and properties. This study aimed to screen metabolite markers with BC diagnosis potentials.
\end{abstract}

Methods: A dried blood spot-based direct infusion mass spectrometry (MS) metabolomic analysis was conducted for $\mathrm{BC}$ and non-BC differentiation. The targeted analytes included 23 amino acids and 26 acylcarnitines.

Results: Multivariate analysis screened out $21 \mathrm{BC}$-related metabolites in the blood. Regression analysis generated a diagnosis model consisting of parameters Pip, Asn, Pro, C14:1/C16, $\mathrm{Phe} / \mathrm{Tyr}$, and Gly/Ala. Tested with another set of BC and non-BC samples, this model showed a sensitivity of $92.2 \%$ and a specificity of $84.4 \%$. Compared to the routinely used protein markers, this model exhibited distinct advantage with its higher sensitivity.

Conclusion: Blood metabolites screening is a more plausible approach for BC detection. Furthermore, this direct MS analysis could be finished within few minutes, which means that its throughput is higher than the currently used imaging techniques.

Keywords: breast cancer, metabolomics, dried blood spot testing

\section{Introduction}

Breast cancer $(\mathrm{BC})$ is the leading cause of cancer-related deaths in women. Although there are many newly emerged screening and therapeutic measures, the morbidity and mortality are still not satisfactorily controlled, posing a great challenge to public health. ${ }^{1}$ Annually, it is estimated that $\sim 1.3$ million new BC cases are diagnosed worldwide. ${ }^{2}$

A growing body of evidence has demonstrated that BC patients' outcome is intensely influenced by cancer stage at the point of diagnosis. Early-stage patients have higher 5 -year survival rate than those diagnosed at later stage. ${ }^{3}$ Thus, effective $\mathrm{BC}$ screening plays key roles in improving survival rates and prognosis. The discovery of BRCA1/2, ERBB2, ESR1/ER, and relevant genes strengthens our ability to discriminate, screen, and treat BC. Unfortunately, the cost and availability of the relevant facilities prevent routine application of genetic screening, especially in the undeveloped countries. Additionally, utility of solely genetic information for early $\mathrm{BC}$ detection is not fully convincing. ${ }^{4}$

Currently, BC screening largely relies on radiologic and serum protein marker detection strategies. ${ }^{5,6}$ Magnetic resonance imaging and computed tomography are relatively reliable tactics that help BC diagnosis clinically, but they are not cost-effective and are not easily accessed by citizens living in the undeveloped regions. ${ }^{7}$ Although mammography checking has claimed to reduce BC-related deaths from $13 \%$ to $25 \%$, it 
is at the expense of $\sim 30 \%$ overdiagnosis in addition to the risk of large-dose radiation exposure ${ }^{8}$ In addition, this screening needs the presence of potential patients in the whole checking process, making it a time-consuming operation.

Blood tumor marker detection has been widely advocated for BC screening, diagnosis, and prognostic prediction. The widely used markers include, but are not limited to, carcinoembryonic antigen (CEA), cancer antigens (CA 27.29, CA 15.3), tissue polypeptide-specific antigen, and tissue polypeptide antigen (TPA). However, these markers lack desired specificity and sensitivity, underscoring the urgent need for alternative simple, accurate, and easy-to-perform screening approaches. ${ }^{9}$

Cancer cells show distinct metabolic characteristics compared to their normal counterparts. Although incomplete, our understanding of metabolic remodeling in cancer cells has been largely reinforced since the discovery of the so-called Warburg effect. ${ }^{10-13}$ The realization of quantitative and qualitative analysis of as many metabolites as possible in a certain system (cell, tissue, or biofluid) in a single run gives birth to the science of metabolomics. Since this conception was firstly coined, metabolomics has been widely used in different aspects of life sciences. The benefited fields include, but are not limited to, disease stratification, biomarker discovery, drug side effect evaluation, and unknown gene function elucidation. $^{14-17}$

Currently prevailing metabolomic techniques mainly include chromatography-mass spectrometry (MS) and nuclear magnetic resonance (NMR) spectroscopy. ${ }^{18}$ The former employs chromatography to separate different metabolites firstly, and then detects the eluted analytes by the MS system hyphenated to it. This mechanism means that the whole analysis process will need more time, implying that its throughput will be limited to some extent. ${ }^{14}$ The latter can directly analyze different raw samples, rendering it a highthroughput feature but usually with low sensitivity. ${ }^{19}$

In BC metabolomic analysis, utilizing gas chromatography-MS system, beta-alanine, 2-hydroyglutarate, glutamate, and xanthine were found increased and glutamine was found decreased in the estrogen receptor-positive BC tissues. ${ }^{20}$ Using an NMR-based serum profiling tactic, histidine, glycerol, acetoacetate, pyruvate, mannose, phenylalanine, and glutamate were reported to be valuable in discriminating patients with metastatic or localized BC. ${ }^{21}$ Unlike blood and tissue metabolomics, urine samples had been explored for screening potential metabolite markers aiding BC diagnosis.2
The entry of MS technology into clinical laboratory was earlier than NMR. The maintenance of MS is easier and cheaper than that of NMR in many aspects. MS-based newborn screening (NS) has been applied for decades. ${ }^{23}$ What should be mentioned specifically is that NS usually employs dried blood spot (DBS) samples collected by heel or finger puncture to simplify the process. Compared to the traditional venous blood samples, the advantages of DBS are that volume of blood taken is much less, they do not need expensive vacuum sample tubes, and they are easy to transport and store. Furthermore, the utilization of direct infusion MS in NS greatly improves the analysis throughput. ${ }^{24}$

Since the DBS-based MS analysis technique can be used for newborn metabolic disorders screening, it might also be useful in tumor metabolite marker screening. In light with this, a DBS-based metabolomic study was performed by using direct infusion MS/MS analysis of BC and the control samples in this study. The quantified metabolites include 23 amino acids and 26 acylcarnitines (Tables S1 and S2), which are commonly encountered in NS. Some ratios based on the above metabolites were also calculated to enrich the analysis information (Table S3). A regression model was then constructed by using parameters that were differentially changed between the two groups. By employing another set of samples, diagnosis ability was evaluated in view of sensitivity and specificity. The purpose of this study was to answer if the DBS-based direct infusion MS technique could be used to facilitate BC screening and diagnosis.

\section{Materials and methods Sample information}

DBS samples from 258 newly diagnosed BC patients and 159 benign mammary gland disease control patients (including 78 healthy people) were collected from The First Affiliated Hospital of Liaoning Medical University. The average ages of BC patients and the controls were 60.4 years (44-80) and 58.7 (42-83), respectively. Statistical analysis showed no age difference between the two groups ( $P=0.351$, Student's $t$-test). The study was approved by the Ethics Committee of The First Affiliated Hospital of Liaoning Medical University. Before DBS collection, written informed consents were acquired from the participants. Of the two groups, metabolomic data from randomly selected 207 patients and 127 age-matched controls ( $\sim 80 \%$ of the total samples) were used as training set to establish regression diagnosis model. The remaining 
$20 \%$ samples were used to evaluate the applicability of the constructed model. All the specimens were fasting blood samples.

\section{Chemicals}

Acetonitrile (high-performance liquid chromatographygrade) and high-purity water were obtained from Thermo Fisher (Waltham, MA, USA). 1-Butanol and acetyl chloride were commercially acquired from Sigma-Aldrich (St Louis, MO, USA). Isotope-labeled internal standards of 12 amino acids (NSK-A) and eight acylcarnitine (NSK-B) from Cambridge Isotope Laboratories (Tewksbury, MA, USA) were used for absolute quantification purpose. All the standards were mixed and dissolved in $2 \mathrm{~mL}$ pure methanol and stored at $4^{\circ} \mathrm{C}$. Working solution was prepared through 100 -fold dilution for metabolite extraction. Amino acids and carnitines quality control (QC) standards were provided by Chromsystems (Grafelfing, Germany). The QC samples were treated as real samples and processed according to the provided instructions to ensure the analysis stability.

\section{Sample preparation}

Except mentioned specifically, all the tests were conducted at room temperature. A $3 \mathrm{~mm}$ (diameter) disc was punched from each DBS paper. The discs were placed into the Millipore MultiScreen HV 96-well plate (Millipore, Billerica, MA, USA) for metabolite extraction. Briefly, every $100 \mu \mathrm{L}$ working solution was added into each well containing a DBS disc. After 20-minute gentle shaking, the plates were centrifuged at $1,500 \times g$ for 2 minutes. The filtrate was collected using new flat-bottom 96-well plates. For each plate, four randomly selected blank wells were added with two low-level and two high-level QC control solutions individually. The QC and filtrate solutions were dried by pure nitrogen gas flow at $50^{\circ} \mathrm{C}$. Dried samples were derivatized at $65^{\circ} \mathrm{C}$ for 20 minutes using $60 \mu \mathrm{L}$ acetyl chloride/1-butanol (10:90, v/v) mixture. The derivatized samples were dried again as mentioned earlier. For metabolomic analysis, each dried sample was dissolved in $100 \mu \mathrm{L}$ fresh mobile phase solution.

\section{Metabolomic analysis}

The direct injection MS metabolomic analysis was conducted by using an AB Sciex 4000 QTrap system (AB Sciex, Framingham, MA, USA). The equipped ion source was electrospray ionization source. All the analytes were scanned under positive mode, and the detailed scan parameters are given in Tables $\mathrm{S} 1$ and $\mathrm{S} 2$.
For each run, every $20 \mu \mathrm{L}$ sample was injected. The mobile phase was $80 \%$ acetonitrile aqueous solution. The initial flow rate was $0.2 \mathrm{~mL} / \mathrm{min}$. Subsequently, the flow rate was reduced to $0.01 \mathrm{~mL} / \mathrm{min}$ within 0.08 minute, kept constant until 1.5 minutes, returned to $0.2 \mathrm{~mL} / \mathrm{min}$ within $0.01 \mathrm{~min}$ ute, and held constant for another 0.5 minute. The ion spray voltage was $4.5 \mathrm{kV}$. Curtain gas pressure was set at 20 psi. A 35 psi pressure was applied to ion source gas 1 and gas 2 . The auxiliary gas temperature was maintained at $350^{\circ} \mathrm{C}$.

Analyst v1.6.0 software (AB Sciex) was used for system control and data collection. ChemoView 2.0.2 (AB Sciex) was used for data preprocessing. Partial least squaresdiscriminant analysis (PLS-DA) was performed by using SIMCA-P v12.0 (Umetrics, Umeå, Sweden). For establishment of BC diagnosis model, binary logistic regression was conducted by using MINITAB v16.0 (Minitab, State College, PA, USA). The diagnostic ability was evaluated by area under the receiver operating characteristic curve. The remaining $20 \%$ samples of each group were used for diagnosis ability appraisal.

\section{Results}

\section{The two groups showed distinct metabolomic difference}

To ensure the method robustness, the QC sample data were firstly evaluated. Detected values from the QC samples all fell into the recommended ranges ( \pm 2 standard deviation), indicating the satisfactory performance of the analysis (data not shown).

For the real samples, a total of 49 metabolites and 22 ratios were detected and calculated for each sample. Using those parameters, a PLS-DA model was established and it showed a clear separation trend between the BC and control groups (Figure 1A). To test if model overfitting has occurred, a permutation test based on 100 iterations was conducted to appraise fitness of the original model against the randomly permuted models. ${ }^{25}$ This operation demonstrated that there was less possibility that the overfitting has occurred in the PLS-DA model (Figure 1B). Thus, the analysis implied that there were really some parameters showing distinct levels between the two groups..$^{25}$

\section{Differential parameter selection}

Using randomly selected $80 \%$ of the $\mathrm{BC}$ and control samples, a multivariate analysis ${ }^{26}$ was carried out to lock potential parameters that had statistic difference between the two groups. It was found that 22 parameters decreased in the 

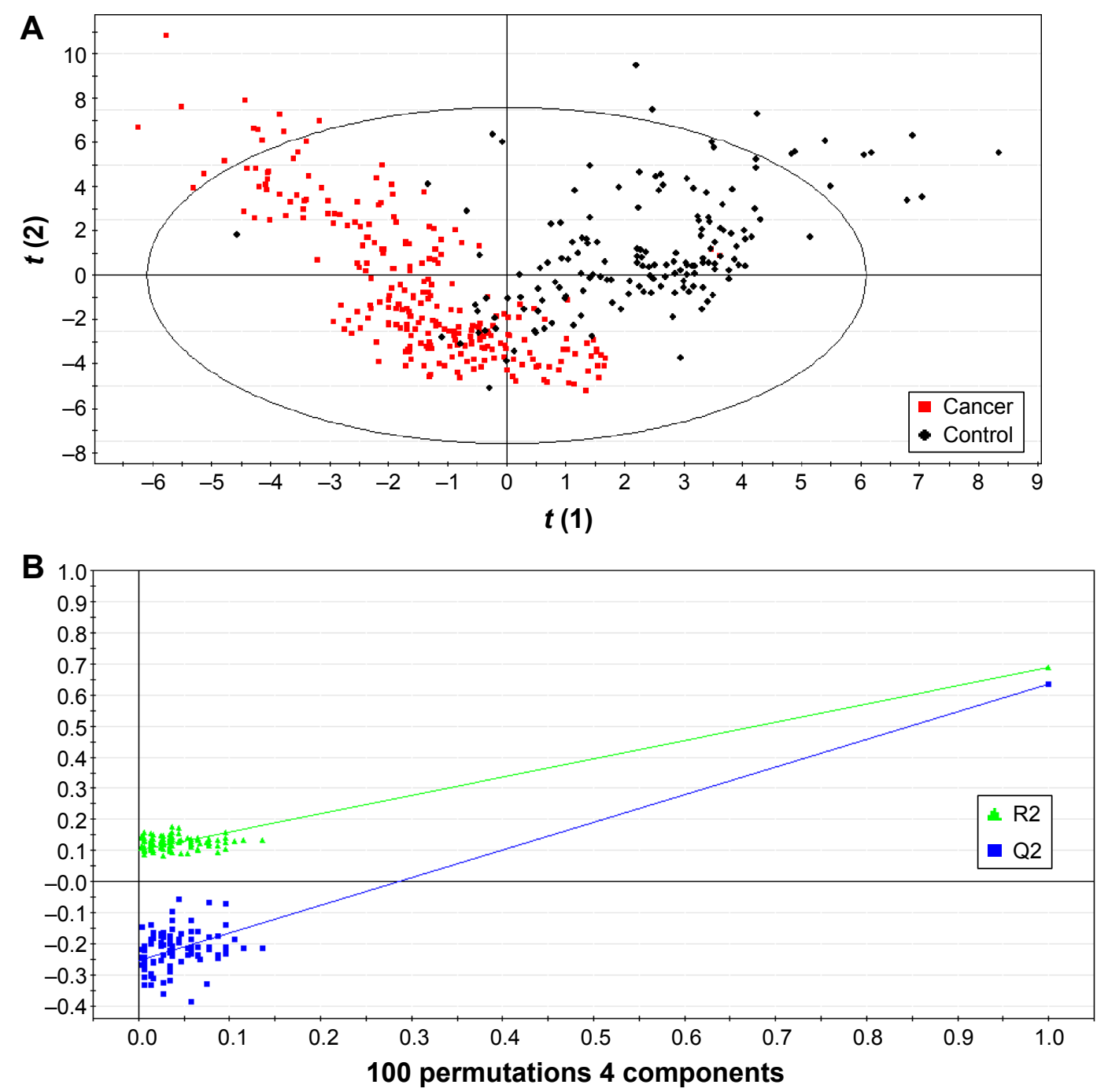

Figure I Partial least squares-discriminant analysis of the metabolomic data.

Notes: (A) Scores plot showing the discrimination between BC and non-BC samples. (B) A I00-time permutation test for validating the corresponding model. The $Y$-axis intercepts were $\mathrm{R}^{2}(0.0,0.10 \mathrm{I})$ and Q2 $(0.0,-0.254)$.

Abbreviation: $\mathrm{BC}$, breast cancer.

$\mathrm{BC}$ group and 13 parameters increased in the $\mathrm{BC}$ group (Figure 2). These variables were further reevaluated by $t$-test, and those of $P$-values $<0.05$ were kept. Finally, 21 parameters were verified to be significantly different between the two groups with only C2, C3, and Tyr increased in the $\mathrm{BC}$ group (Table 1).

\section{Diagnostic regression equation}

In order to test if the 21 differential parameters could be used for $\mathrm{BC}$ diagnosis purpose, a binary logistic regression analysis was conducted. The final equation contained only three amino acids and three calculated ratios, Pip, Asn, Pro, C14:1/C16, Phe/Tyr, and Gly/Ala (Figure 3). Receiver operating characteristic evaluating the diagnosis model gave a sensitivity and specificity of $90.3 \%$ and $87.4 \%$, respectively, when the cutoff was set to 4.8754 (Figure 4). Area under the curve was 0.944 . Further tested by using each remaining $20 \%$ samples of each group, the model gave a diagnosis sensitivity of $92.16 \%$ and a specificity of $84.38 \%$.

\section{Discussion}

One of the major challenges for successful diagnosis and treatment of $\mathrm{BC}$ is the lack of reliable molecular predictors. Over the past decades, there have been a rapidly growing number of metabolomic researches aimed at finding biomarkers that could be used to aid $\mathrm{BC}$ diagnosis, evaluate response to therapy, and provide treatment guidance. ${ }^{27,28}$

Although tumor cells show distinct metabolic features, they really share the same metabolic pathways and metabolites with their normal counterparts. Thus, we performed a 


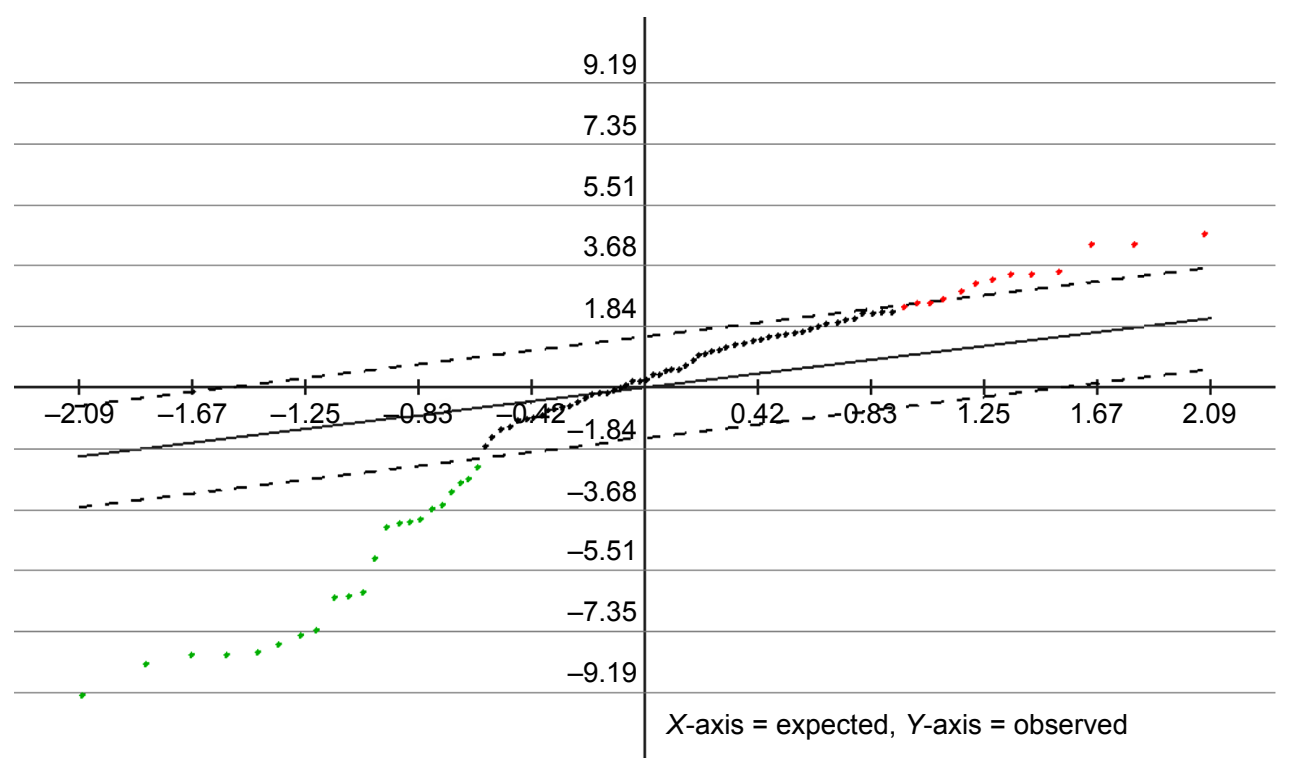

Figure 2 SAM analysis results with the false discovery rate set to zero.

Notes: Points (metabolites or ratios) above (elevated in BC group) or under (decreased in BC group) the dashed lines were those changed significantly. Abbreviations: BC, breast cancer; SAM, significance analysis of microarrays.

DBS-based metabolomic assay aimed at amino acids and acylcarnitines, attempted to find valuable clues to help BC diagnosis.

Amino acids are the basic building blocks for nearly all cell types. In the context of cancer metabolomics, many

Table I Differential parameters between BC and control groups identified using the training set data

\begin{tabular}{|c|c|c|c|c|c|}
\hline No & Parameters & Status* & $P$-value & $\begin{array}{l}\text { Control } \\
\text { mean } \pm \text { SD }\end{array}$ & $\begin{array}{l}\text { BC } \\
\text { mean } \pm \text { SD }\end{array}$ \\
\hline I & $\mathrm{C} 3$ & $\uparrow$ & 0.000 & $1.4 \pm 0.7$ & $2.1 \pm 1.3$ \\
\hline 2 & Tyr & $\uparrow$ & 0.001 & $42.6 \pm 12.5$ & $51.0 \pm 16.8$ \\
\hline 3 & $\mathrm{C} 2$ & $\uparrow$ & 0.000 & $|3| \pm 8.0$. & $19.5 \pm 10.8$ \\
\hline 4 & Cys & $\downarrow$ & 0.000 & $1.2 \pm 1.0$ & $0.44 \pm 0.45$ \\
\hline 5 & Pro & $\downarrow$ & 0.000 & $308.2 \pm 175.2$ & $194.7 \pm 82.5$ \\
\hline 6 & Asn & $\downarrow$ & 0.000 & $71.0 \pm 28.1$ & $47.6 \pm 25.1$ \\
\hline 7 & Pip & $\downarrow$ & 0.000 & $352.1 \pm 452.8$ & $97.0 \pm 97.3$ \\
\hline 8 & Hcy & $\downarrow$ & 0.000 & $6.9 \pm 1.6$ & $5.8 \pm 0.9$ \\
\hline 9 & Trp & $\downarrow$ & 0.000 & $47.1 \pm 21.1$ & $28.8 \pm 20.7$ \\
\hline 10 & $\mathrm{CI} 4: 2$ & $\downarrow$ & 0.000 & $5.1 \pm 7.7$ & $0.9 \pm 2.0$ \\
\hline II & $\mathrm{Cl0} 2 / \mathrm{ClO}$ & $\downarrow$ & 0.000 & $5.2 \pm 3.9$ & $2.4 \pm 2.6$ \\
\hline 12 & $\mathrm{ClO}: 2$ & $\downarrow$ & 0.000 & $0.3 \pm 0.2$ & $0.2 \pm 0.1$ \\
\hline 13 & Phe/Tyr & $\downarrow$ & 0.000 & $1.2 \pm 0.3$ & $1.0 \pm 0.2$ \\
\hline 14 & $\mathrm{Cit} / \mathrm{Arg}$ & $\downarrow$ & 0.002 & $6.1 \pm 5.5$ & $3.3 \pm 3.3$ \\
\hline 15 & Lys & $\downarrow$ & 0.000 & $72.2 \pm 121.9$ & $25.5 \pm 40.9$ \\
\hline 16 & $\mathrm{CI} 8: 1-\mathrm{OH}$ & $\downarrow$ & 0.001 & $2.7 \pm 5.5$ & $1.0 \pm 2.0$ \\
\hline 17 & His & $\downarrow$ & 0.000 & $57.3 \pm 134.8$ & $19.0 \pm 23.9$ \\
\hline 18 & Tyr/Cit & $\downarrow$ & 0.011 & $0.9 \pm 0.4$ & $0.7 \pm 0.3$ \\
\hline 19 & $\mathrm{Cl} 4: \mathrm{I} / \mathrm{Cl} 6$ & $\downarrow$ & 0.000 & $0.9 \pm 0.5$ & $0.2 \pm 0.3$ \\
\hline 20 & $\mathrm{Cl} 8-\mathrm{OH}$ & $\downarrow$ & 0.002 & $1.6 \pm 4.6$ & $0.6 \pm 1.3$ \\
\hline 21 & Gly/Ala & $\downarrow$ & 0.001 & $157.1 \pm 69.4$ & I54.3 $\pm 48 . \mid$ \\
\hline
\end{tabular}

Note: *Compared to the control group.

Abbreviations: BC, breast cancer; SD, standard deviation. amino acids have been demonstrated to provide valuable clues for studying pathogenesis and to act as potential indicators for diverse malignancies. ${ }^{29}$ Specific plasma amino acid changes have been reported in patients suffering from breast, lung, and head and neck cancers through metabolomic analysis. $^{30,31}$

Cascino et al found that Orn, Glu, and Trp increased in the patient plasma. ${ }^{30}$ Miyagi et al demonstrated that Gln, His, Trp, Tyr, and Phe decreased in the plasma, whereas Gly, Ala, Pro, and Thr increased. ${ }^{32}$ In this study, only Tyr increased in BC blood, but Cys, Pro, Asn, Pip, Hcy, Trp, Lys, and His decreased (Table 1). This discrepancy might be due to the fact that only in this study, the control group included the benign mammary gland diseases and the healthy people simultaneously. Except the disagreement among these studies, Tyr deficiency had been demonstrated to result in BC cell growth arrest. ${ }^{33}$ Thus, increased blood Tyr might do favor to BC. Of note, blood Tyr is affected by diet. It cannot be de novo synthesized by human body. It is also not easy to exclude the possibility that increased Tyr might be the metabolic adaptation to tumor state.

Except Tyr, there were eight decreased amino acids in the $\mathrm{BC}$ blood. It was most likely that such phenomena were the result of excessive consumption of amino acids by $\mathrm{BC}$ tissues. As what has been well accepted, tumor cells need more amino acids to sustain their uncontrolled growth. ${ }^{34}$

Carnitine plays key roles in fatty acids catabolism. It acts as a shutter to bring fatty acids into mitochondria for 

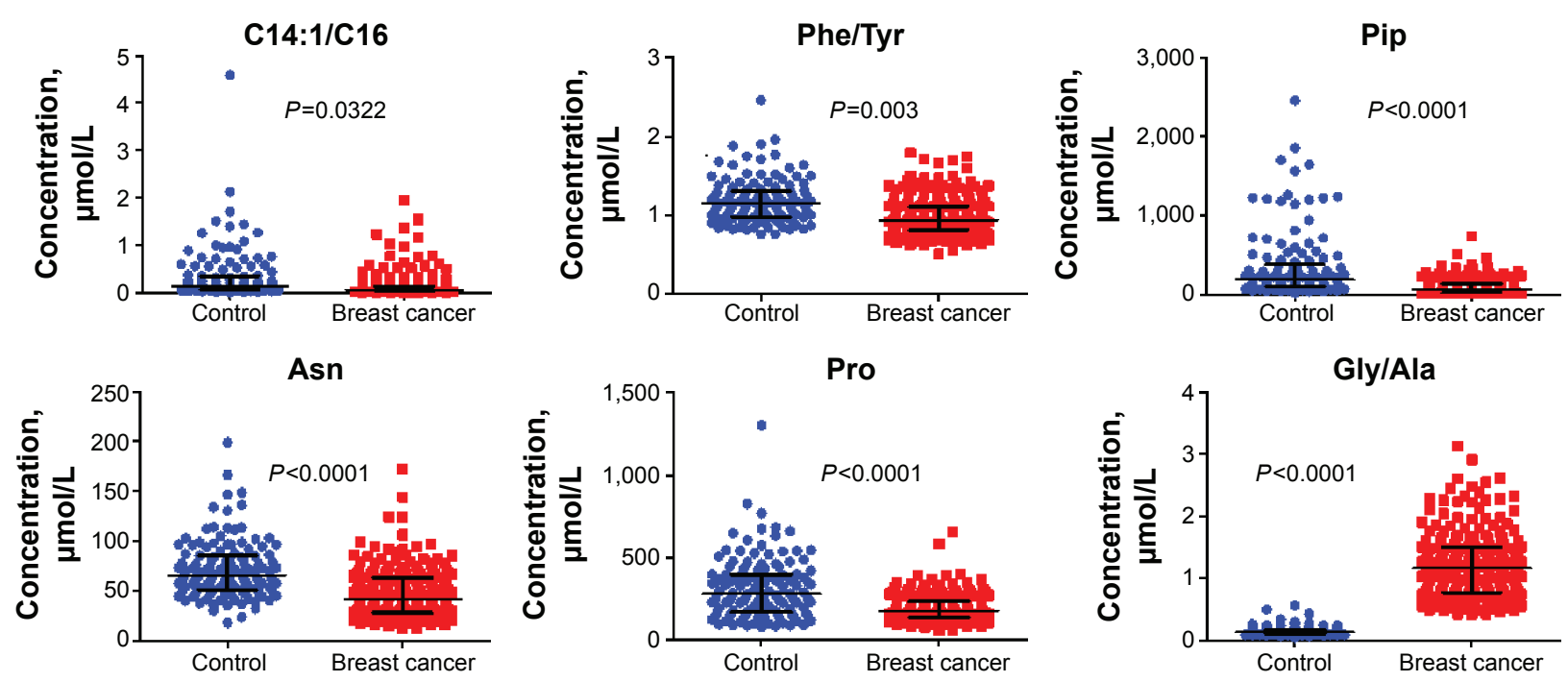

Figure 3 Levels of six metabolites included in the regression model.

oxidation. A clear trend in this study was that short-chain carnitine increased in BC plasma (Table 1). In a study of myeloma, C2 carnitine was identified as a potential biomarker to indicate disease activity and relapse. ${ }^{35}$ Increased $\mathrm{C} 2$ carnitine was also reported in the urine of patients suffering from hepatocellular carcinoma. ${ }^{36}$ Also in hepatocellular carcinoma, C3 carnitine was found at high level in cancer tissues. ${ }^{37}$ Although there was no evidence to correlate short-chain carnitines with $\mathrm{BC}$, they were more likely to be potential markers to indicate the occurrence of many malignancies.

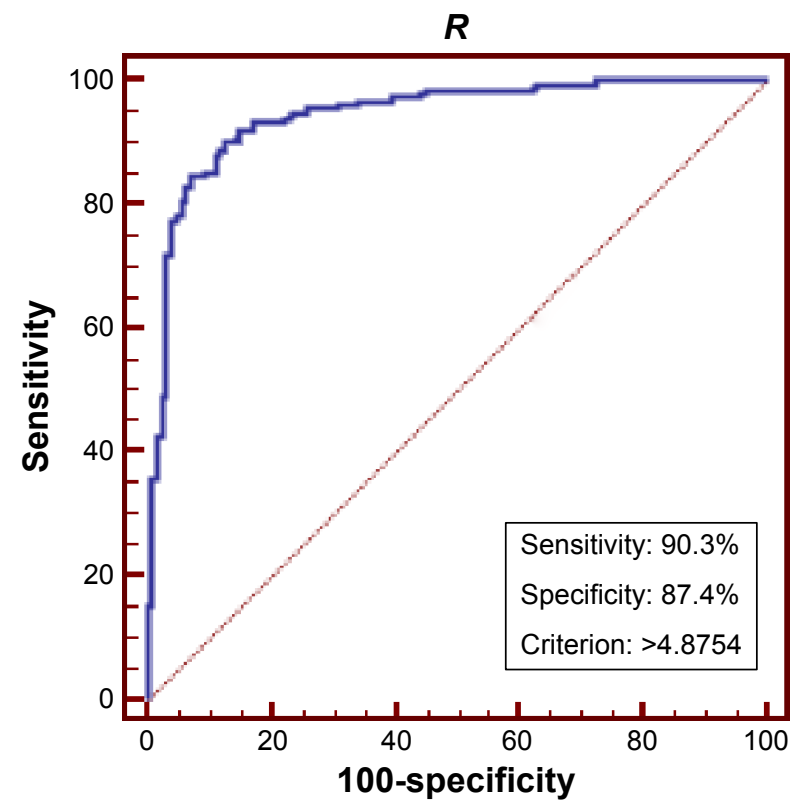

Figure 4 ROC based on the regression model.

Note: Model equation was $y=-\mathrm{Cl} 4: \mathrm{I} / \mathrm{Cl} 6 \times 4.24-\mathrm{Phe} / \mathrm{Tyr} \times 3.32-\mathrm{Pip} \times 0.0 \mathrm{I}-$ Asn $\times 0.05-$ Pro $\times 0.01$ - Gly/Ala $\times 3.11+14.91$.

Abbreviations: ROC, receiver operating characteristic; $\mathrm{R}$, regression result.
In order to explore the utility of the differentially expressed metabolites, a regression model was constructed by using parameters listed in Table 1 . The result showed that combined use of six parameters could appropriately differentiate $\mathrm{BC}$ from non-BC samples (Figures 3 and 4). Among them, only one ratio, the carnitines $\mathrm{C} 14: 1 / \mathrm{C} 16$, was included. Increased C14:1/C16 acylcarnitine ratio was closely linked to impaired mitochondria fatty acid beta-oxidation, ${ }^{38}$ coinciding with the fact that mitochondrial impairment had been demonstrated in $\mathrm{BC}$ cells. ${ }^{39}$

In the regression model, Pip, Asn, and Pro were the only included free amino acids. Their levels were all decreased. We speculated that the decrease was more likely due to the excessive consumption by tumor cells. Besides the free amino acids, elevated Phe/Tyr and decreased Gly/Ala were closely related to the differentiation of $\mathrm{BC}$ and non-BC (Figure 3). High ratio of Phe/Tyr had been described in malignant histiocytosis and some other cancerous diseases. ${ }^{40,41}$ As of the decreased Gly/Ala ratio, it was most possible owing to the fact that rapid proliferating tumor cells need more Gly. ${ }^{34}$

Although the diagnosis specificity (87.4\%) of the regression model was not comparable with that of CA 15.3 (93\%-95\%), CEA (45\%-95\%), and TPA ( 81\%), the sensitivity $(90.3 \%)$ was superior to that of CA $15.3(44 \%-64 \%)$, CEA $(\sim 45 \%)$, and TPA $(\sim 67 \%))^{42,43}$ Thus, for BC screening purpose, the DBS-based MS technique was a promising tool for early BC discovery.

\section{Conclusion}

In summary, a proper diagnostic model with only six blood parameters was established and could be utilized to 
discriminate $\mathrm{BC}$ from non-BC. It could be expected that the DBS-based MS strategy was a promising alternative for BC screening because of its higher sensitivity. Additionally, the whole MS analysis could be completed within few minutes. Unfortunately, the traditional plasma protein tumor markers were not simultaneously measured for these specimens; therefore, the diagnosis ability of combined use of traditional markers and the metabolite markers could not be evaluated. Further analysis should be addressed to the relevant topics to ascertain the exact value of DBS-based MS metabolomic analysis in BC screening and diagnosis.

\section{Acknowledgments}

This study was partially supported by the National Natural Science Foundation of China (No 81372695) and by the Department of Science and Technology of Liaoning Province (LNCCC-D27-2015).

\section{Disclosure}

The authors declare no conflicts of interest in this work.

\section{References}

1. Abd El-Rahman AA, El-Shafei SM, Ivanova EV, et al. Cytotoxicity of Trichoderma spp. cultural filtrate against human cervical and breast cancer cell lines. Asian Pac J Cancer Prev. 2014;15(17):7229-7234.

2. Jemal A, Bray F, Center MM, et al. Global cancer statistics. CA Cancer J Clin. 2011;61(2):69-90.

3. Zhang A, Sun H, Wang X. Serum metabolomics as a novel diagnostic approach for disease: a systematic review. Anal Bioanal Chem. 2012 404(4):1239-1245.

4. Kang E, Seong MW, Park SK, et al. The prevalence and spectrum of BRCA1 and BRCA2 mutations in Korean population: recent update of the Korean Hereditary Breast Cancer (KOHBRA) study. Breast Cancer Res Treat. 2015;151(1):157-168.

5. Shi L. Racial differences in breast cancer patterns. Science. 2010; 329(5987):32.

6. Possemato R, Marks KM, Shaul YD, et al. Functional genomics reveal that the serine synthesis pathway is essential in breast cancer. Nature. 2011;476(7360):346-350.

7. Luczynska E, Heinze-Paluchowska S, Hendrick E, et al. Comparison between breast MRI and contrast-enhanced spectral mammography. Med Sci Monit. 2015;21:1358-1367.

8. Loberg M, Lousdal ML, Bretthauer M, et al. Benefits and harms of mammography screening. Breast Cancer Res. 2015;17(1):63.

9. Asiago VM, Alvarado LZ, Shanaiah N, et al. Early detection of recurrent breast cancer using metabolite profiling. Cancer Res. 2010;70(21): 8309-8318.

10. Hanahan D, Weinberg RA. The hallmarks of cancer. Cell. 2000;100(1): $57-70$.

11. Locasale JW, Vander Heiden MG, Cantley LC. Rewiring of glycolysis in cancer cell metabolism. Cell Cycle. 2010;9(21):4253.

12. Vander Heiden MG, Locasale JW, Swanson KD, et al. Evidence for an alternative glycolytic pathway in rapidly proliferating cells. Science. 2010;329(5998):1492-1499.

13. Locasale JW, Cantley LC. Altered metabolism in cancer. BMC Biol. 2010;8:88

14. Gowda GA, Djukovic D. Overview of mass spectrometry-based metabolomics: opportunities and challenges. Methods Mol Biol. 2014; 1198:3-12.
15. Wang X, Xie Y, Gao P, et al. A metabolomics-based method for studying the effect of $y f c C$ gene in Escherichia coli on metabolism. Anal Biochem. 2014;451:48-55.

16. Wojakowska A, Chekan M, Widlak P, et al. Application of metabolomics in thyroid cancer research. Int J Endocrinol. 2015;2015:258763.

17. Chahine LM, Stern MB, Chen-Plotkin A. Blood-based biomarkers for Parkinson's disease. Parkinsonism Relat Disord. 2014;20 Suppl 1: S99-S103.

18. Gowda GA, Zhang S, Gu H, et al. Metabolomics-based methods for early disease diagnostics. Expert Rev Mol Diagn. 2008;8(5):617-633.

19. Bathen TF, Sitter B, Sjobakk TE, et al. Magnetic resonance metabolomics of intact tissue: a biotechnological tool in cancer diagnostics and treatment evaluation. Cancer Res. 2010;70(17): 6692-6696.

20. Budczies J, Brockmoller SF, Muller BM, et al. Comparative metabolomics of estrogen receptor positive and estrogen receptor negative breast cancer: alterations in glutamine and beta-alanine metabolism. J Proteomics. 2013;94:279-288.

21. Jobard E, Pontoizeau C, Blaise BJ, et al. A serum nuclear magnetic resonance-based metabolomic signature of advanced metastatic human breast cancer. Cancer Lett. 2014;343(1):33-41.

22. Kim Y, Koo I, Jung BH, et al. Multivariate classification of urine metabolome profiles for breast cancer diagnosis. BMC Bioinformatics. 2010;11 Suppl 2:S4.

23. Atkinson K, Zuckerman B, Sharfstein JM, et al. A public health response to emerging technology: expansion of the Massachusetts newborn screening program. Public Health Rep. 2001;116(2):122-131.

24. Ombrone D, Giocaliere E, Forni G, et al. Expanded newborn screening by mass spectrometry: new tests, future perspectives. Mass Spectrom Rev. 2016;35(1):71-84.

25. Huang Z, Lin L, Gao Y, et al. Bladder cancer determination via two urinary metabolites: a biomarker pattern approach. Mol Cell Proteomics. 2011;10(10):M111.

26. Tusher VG, Tibshirani R, Chu G. Significance analysis of microarrays applied to the ionizing radiation response. Proc Natl Acad Sci US A. 2001; 98(9):5116-5121.

27. Curtis C, Shah SP, Chin SF, et al. The genomic and transcriptomic architecture of 2,000 breast tumours reveals novel subgroups. Nature. 2012;486(7403):346-352.

28. Shah SP, Roth A, Goya R, et al. The clonal and mutational evolution spectrum of primary triple-negative breast cancers. Nature. 2012;486(7403):395-399.

29. Kimura T, Noguchi Y, Shikata N, et al. Plasma amino acid analysis for diagnosis and amino acid-based metabolic networks. Curr Opin Clin Nutr Metab Care. 2009;12(1):49-53.

30. Cascino A, Muscaritoli M, Cangiano C, et al. Plasma amino acid imbalance in patients with lung and breast cancer. Anticancer Res. 1995;15(2):507-510.

31. Kubota A, Meguid MM, Hitch DC. Amino acid profiles correlate diagnostically with organ site in three kinds of malignant tumors. Cancer. 1992;69(9):2343-2348.

32. Miyagi Y, Higashiyama M, Gochi A, et al. Plasma free amino acid profiling of five types of cancer patients and its application for early detection. PLoS One. 2011;6(9):e24143.

33. Harvie MN, Campbell IT, Howell A, et al. Acceptability and tolerance of a low tyrosine and phenylalanine diet in patients with advanced cancer - a pilot study. J Hum Nutr Diet. 2002;15(3):193-202.

34. Jain M, Nilsson R, Sharma S, et al. Metabolite profiling identifies a key role for glycine in rapid cancer cell proliferation. Science. 2012; 336(6084):1040-1044.

35. Lodi A, Tiziani S, Khanim FL, et al. Proton NMR-based metabolite analyses of archived serial paired serum and urine samples from myeloma patients at different stages of disease activity identifies acetylcarnitine as a novel marker of active disease. PLoS One. 2013;8(2): e56422.

36. Ladep NG, Dona AC, Lewis MR, et al. Discovery and validation of urinary metabotypes for the diagnosis of hepatocellular carcinoma in West Africans. Hepatology. 2014;60(4):1291-1301. 
37. Huang Q, Tan Y, Yin P, et al. Metabolic characterization of hepatocellular carcinoma using nontargeted tissue metabolomics. Cancer Res. 2013;73(16):4992-5002.

38. Pooya S, Blaise S, Moreno GM, et al. Methyl donor deficiency impairs fatty acid oxidation through PGC-1alpha hypomethylation and decreased ER-alpha, ERR-alpha, and HNF-4alpha in the rat liver. J Hepatol. 2012;57(2):344-351.

39. Putignani L, Raffa S, Pescosolido R, et al. Preliminary evidences on mitochondrial injury and impaired oxidative metabolism in breast cancer. Mitochondrion. 2012;12(3):363-369.

40. Neurauter G, Grahmann AV, Klieber M, et al. Serum phenylalanine concentrations in patients with ovarian carcinoma correlate with concentrations of immune activation markers and of isoprostane-8. Cancer Lett. 2008;272(1):141-147.
41. Imashuku S, Morioka Y, Kodo N, et al. Abnormal serum phenylalaninetyrosine ratio and hyperferritinemia in malignant histiocytosis. Pediatr Hematol Oncol. 1988;5(4):299-307.

42. Vizcarra E, Lluch A, Cibrian R, et al. Value of CA 15.3 in breast cancer and comparison with CEA and TPA: a study of specificity in diseasefree follow-up patients and sensitivity in patients at diagnosis of the first metastasis. Breast Cancer Res Treat. 1996;37(3):209-216.

43. Zheng H, Luo RC. Diagnostic value of combined detection of TPS, CA153 and CEA in breast cancer. Di Yi Jun Yi Da Xue Xue Bao. 2005; 25(10):1293-1294, 1298 


\section{Supplementary materials}

Table SI The amino acids detected, corresponding scan modes, equipment settings, and quantification IS used in the study

\begin{tabular}{|c|c|c|c|c|c|c|c|c|c|c|c|c|}
\hline No & Abbreviation & Full name & Scan type & $\begin{array}{l}\text { Loss } \\
(\mathrm{Da})\end{array}$ & $\begin{array}{l}\text { Start-stop } \\
\text { (Da) }\end{array}$ & $\begin{array}{l}\text { Fragment } \\
\text { transition }\end{array}$ & DP (V) & EP (V) & CE (V) & $\operatorname{CXP}(V)$ & IS & IS $(\mu \mathrm{M})$ \\
\hline I & Ala & Alanine & Neutral loss & 102.1 & $130-280$ & & 40 & 10 & 19 & 3 & d4-Ala & 2.5 \\
\hline 2 & $\operatorname{Arg}$ & Arginine & MRM & & & $231.2 \rightarrow 70.0$ & 55 & 10 & 41 & 2 & d5-Arg & 2.5 \\
\hline 3 & Asn & Asparagine & Neutral loss & 102.1 & $130-280$ & & 40 & 10 & 19 & 3 & d3-Leu & 2.5 \\
\hline 4 & Asp & Aspartate & Neutral loss & 102.1 & $130-280$ & & 40 & 10 & 19 & 3 & d3-Asp & 2.5 \\
\hline 5 & Cit & Citrulline & MRM & & & $232.2 \rightarrow|| 3.1$ & 44 & 10 & 25 & 2 & d2-Cit & 2.5 \\
\hline 6 & Cys & Cysteine & Neutral loss & 102.1 & $130-280$ & & 40 & 10 & 19 & 3 & d8-Val & 2.5 \\
\hline 7 & Gln & Glutamine & Neutral loss & 102.1 & $130-280$ & & 40 & 10 & 19 & 3 & d3-Met & 2.5 \\
\hline 8 & Glu & Glutamic & Neutral loss & 102.1 & $130-280$ & & 40 & 10 & 19 & 3 & d3-Glu & 2.5 \\
\hline 9 & Gly & Glycine & MRM & & & $132 . \mid \rightarrow 76.0$ & 36 & 10 & 14 & 2 & I5NI3C-Gly & 12.5 \\
\hline 10 & Hcy & Homocysteine & Neutral loss & 102.1 & $130-280$ & & 40 & 10 & 19 & 3 & d3-Leu & 2.5 \\
\hline II & $\mathrm{His}$ & Histidine & Neutral loss & 102.1 & $130-280$ & & 40 & 10 & 19 & 3 & d3-Met & 2.5 \\
\hline 12 & Leu & Leucine & Neutral loss & 102.1 & $130-280$ & & 40 & 10 & 19 & 3 & d3-Leu & 2.5 \\
\hline 13 & Lys & Lysine & Neutral loss & 102.1 & $130-280$ & & 40 & 10 & 19 & 3 & d3-Met & 2.5 \\
\hline 14 & Met & Methionine & Neutral loss & 102.1 & $130-280$ & & 40 & 10 & 19 & 3 & d3-Met & 2.5 \\
\hline 15 & Orn & Ornithine & MRM & & & $189.2 \rightarrow 70.1$ & 37 & 10 & 34 & 2 & d2-Orn & 2.5 \\
\hline 16 & Phe & Phenylalanine & Neutral loss & 102.1 & $130-280$ & & 40 & 10 & 19 & 3 & d6-Phe & 2.5 \\
\hline 17 & Pip & Piperamide & Neutral loss & 102.1 & $130-280$ & & 40 & 10 & 19 & 3 & d8-Val & 2.5 \\
\hline 18 & Pro & Proline & Neutral loss & 102.1 & $130-280$ & & 40 & 10 & 19 & 3 & d8-Val & 2.5 \\
\hline 19 & Ser & Serine & Neutral loss & 102.1 & $130-280$ & & 40 & 10 & 19 & 3 & d4-Ala & 2.5 \\
\hline 20 & Thr & Threonine & Neutral loss & 102.1 & $130-280$ & & 40 & 10 & 19 & 3 & d8-Val & 2.5 \\
\hline 21 & $\operatorname{Trp}$ & Tryptophan & Neutral loss & 102.1 & $130-280$ & & 40 & 10 & 19 & 3 & d3-Glu & 2.5 \\
\hline 22 & Tyr & Tyrosine & Neutral loss & 102.1 & $130-280$ & & 40 & 10 & 19 & 3 & $13 \mathrm{C} 6-\mathrm{Tyr}$ & 2.5 \\
\hline 23 & Val & Valine & Neutral loss & 102.1 & $130-280$ & & 40 & 10 & 19 & 3 & d8-Val & 2.5 \\
\hline
\end{tabular}

Abbreviations: IS, internal standard; DP, declustering potential; EP, entrance potential; CE, collision energy; CXP, collision cell exit potential; MRM, multiple reaction monitoring.

Table S2 The precursor scan mode-detected carnitines, corresponding equipment settings, and quantification IS used in the study

\begin{tabular}{|c|c|c|c|c|c|c|c|c|c|c|}
\hline No & Abbreviation & Full name & $\begin{array}{l}\text { Start-stop } \\
\text { (Da) }\end{array}$ & $\begin{array}{l}\text { Precursor } \\
(\mathrm{m} / \mathrm{z})\end{array}$ & DP (V) & $E P(V)$ & CE (V) & CXP (V) & IS & IS $(\mu \mathrm{M})$ \\
\hline I & $\mathrm{CO}$ & Free carnitine & $210-610$ & 85.1 & $40-75$ & 10 & $35-55$ & 3 & $\mathrm{~d} 9-\mathrm{CO}$ & 0.76 \\
\hline 2 & $\mathrm{C} 2$ & Acetylcarnitine & $210-610$ & 85.1 & $40-75$ & 10 & $35-55$ & 3 & $\mathrm{~d} 3-\mathrm{C2}$ & 0.19 \\
\hline 3 & $\mathrm{C} 3$ & Propionylcarnitine & $210-610$ & 85.1 & $40-75$ & 10 & $35-55$ & 3 & d3-C3 & 0.04 \\
\hline 4 & $\mathrm{C} 4$ & Butyrylcarnitine & $210-610$ & 85.1 & $40-75$ & 10 & $35-55$ & 3 & $\mathrm{~d} 3-\mathrm{C} 4$ & 0.04 \\
\hline 5 & $\mathrm{C} 4 \mathrm{OH}$ & 3-Hydroxylbutyrylcarnitine & $210-610$ & 85.1 & $40-75$ & 10 & $35-55$ & 3 & $\mathrm{~d} 9-\mathrm{C5}$ & 0.04 \\
\hline 6 & C4DC & Succinyl-/methylmalonylcarnitine & $210-610$ & 85.1 & $40-75$ & 10 & $35-55$ & 3 & $\mathrm{~d} 3-\mathrm{C} 8$ & 0.04 \\
\hline 7 & C5 & Cisovalerylcarnitine & $210-610$ & 85.1 & $40-75$ & 10 & $35-55$ & 3 & d9-C5 & 0.04 \\
\hline 8 & $\mathrm{C} 5-\mathrm{OH}$ & 3-Hydroxyisovalerylcarnitine & $210-610$ & 85.1 & $40-75$ & 10 & $35-55$ & 3 & $\mathrm{~d} 9-\mathrm{C5}$ & 0.04 \\
\hline 9 & C5DC & Glutarylcarnitine & $210-610$ & 85.1 & $40-75$ & 10 & $35-55$ & 3 & $\mathrm{~d} 3-\mathrm{C} 8$ & 0.04 \\
\hline 10 & C5:I & Tiglylcarnitine & $210-610$ & 85.1 & $40-75$ & 10 & $35-55$ & 3 & d9-C5 & 0.04 \\
\hline 11 & $\mathrm{C} 6$ & Hexanoylcarnitine & $210-610$ & 85.1 & $40-75$ & 10 & $35-55$ & 3 & $\mathrm{~d} 9-\mathrm{C5}$ & 0.04 \\
\hline 12 & $\mathrm{C} 8$ & Octanoylcarnitine & $210-610$ & 85.1 & $40-75$ & 10 & $35-55$ & 3 & $\mathrm{~d} 3-\mathrm{C} 8$ & 0.04 \\
\hline 13 & $\mathrm{ClO}$ & Decanoylcarnitine & $210-610$ & 85.1 & $40-75$ & 10 & $35-55$ & 3 & $\mathrm{~d} 3-\mathrm{C} 8$ & 0.04 \\
\hline 14 & $\mathrm{Cl} 2$ & Lauroylcarnitine & $210-610$ & 85.1 & $40-75$ & 10 & $35-55$ & 3 & d9-Cl4 & 0.04 \\
\hline 15 & $\mathrm{Cl} 4$ & Myristoylcarnitine & $210-610$ & 85.1 & $40-75$ & 10 & $35-55$ & 3 & d9-Cl4 & 0.04 \\
\hline 16 & $\mathrm{Cl} 4-\mathrm{OH}$ & 3-Hydroxyl-tetradecanoylcarnitine & $210-610$ & 85.1 & $40-75$ & 10 & $35-55$ & 3 & d9-Cl4 & 0.04 \\
\hline 17 & CI4DC & Tetradecanoyldiacylcarnitine & $210-610$ & 85.1 & $40-75$ & 10 & $35-55$ & 3 & $\mathrm{~d} 3-\mathrm{Cl} 6$ & 0.08 \\
\hline 18 & $\mathrm{CI} 4: \mathrm{I}$ & Tetradecenoylcarnitine & $210-610$ & 85.1 & $40-75$ & 10 & $35-55$ & 3 & d9-Cl4 & 0.04 \\
\hline 19 & $\mathrm{Cl} 6$ & Palmitoylcarnitine & $210-610$ & 85.1 & $40-75$ & 10 & $35-55$ & 3 & $\mathrm{~d} 3-\mathrm{Cl} 6$ & 0.08 \\
\hline 20 & $\mathrm{Cl} 6-\mathrm{OH}$ & 3-Hydroxypalmitoylcarnitine & $210-610$ & 85.1 & $40-75$ & 10 & $35-55$ & 3 & $\mathrm{~d} 3-\mathrm{Cl} 6$ & 0.08 \\
\hline 21 & $\mathrm{Cl} 6: \mathrm{I}-\mathrm{OH}$ & 3-Hydroxypalmitoleylcarnitine & $210-610$ & 85.1 & $40-75$ & 10 & $35-55$ & 3 & $\mathrm{~d} 3-\mathrm{Cl} 6$ & 0.08 \\
\hline 22 & $\mathrm{Cl} 8$ & Octadecanoylcarnitine & $210-610$ & 85.1 & $40-75$ & 10 & $35-55$ & 3 & $\mathrm{~d} 3-\mathrm{Cl} 6$ & 0.08 \\
\hline 23 & $\mathrm{C} 20$ & Arachidic carnitine & $210-610$ & 85.1 & $40-75$ & 10 & $35-55$ & 3 & $\mathrm{~d} 3-\mathrm{Cl} 6$ & 0.08 \\
\hline 24 & $\mathrm{C} 22$ & Behenic carnitine & $210-610$ & 85.1 & $40-75$ & 10 & $35-55$ & 3 & $\mathrm{~d} 3-\mathrm{Cl} 6$ & 0.08 \\
\hline 25 & $\mathrm{C} 24$ & Tetracosanoic carnitine & $210-610$ & 85.1 & $40-75$ & 10 & $35-55$ & 3 & $\mathrm{~d} 3-\mathrm{Cl} 6$ & 0.08 \\
\hline 26 & $\mathrm{C} 26$ & Hexacosanoic carnitine & $210-610$ & 85.1 & $40-75$ & 10 & $35-55$ & 3 & $\mathrm{~d} 3-\mathrm{Cl} 6$ & 0.08 \\
\hline
\end{tabular}

Abbreviations: IS, internal standard; DP, declustering potential; EP, entrance potential; CE, collision energy; CXP, collision cell exit potential; $\mathrm{m} / \mathrm{z}$, mass/charge ratio. 
Table S3 Parameters derived from the quantified metabolites

\begin{tabular}{|c|c|c|c|}
\hline$\overline{N o}$ & Name & No & Name \\
\hline 1 & Arg/Orn & 23 & C5DC/CI6 \\
\hline 2 & Cit/Arg & 24 & $\mathrm{C} 8 / \mathrm{C} 2$ \\
\hline 3 & Gly/Ala & 25 & $\mathrm{C} 8 / \mathrm{ClO}$ \\
\hline 4 & Met/Leu & 26 & $\mathrm{Cl} 6-\mathrm{OH} / \mathrm{Cl} 6$ \\
\hline 5 & Met/Phe & 27 & $\mathrm{C} 26 / \mathrm{C} 20$ \\
\hline 6 & Orn/Cit & 28 & $\mathrm{Cl} 4: 1 / \mathrm{Cl} 6$ \\
\hline 7 & Phe/Tyr & 29 & C3DC \\
\hline 8 & Tyr/Cit & 30 & C3DC/CIO \\
\hline 9 & Val/Phe & 31 & CI8:I \\
\hline 10 & $\mathrm{C} 2 / \mathrm{CO}$ & 32 & $\mathrm{Cl} 8-\mathrm{OH}$ \\
\hline II & $\mathrm{C} 3 / \mathrm{CO}$ & 33 & CI8:I-OH \\
\hline 12 & $\mathrm{C} 3 / \mathrm{C} 2$ & 34 & CIO:I \\
\hline 13 & $\mathrm{C} 3 / \mathrm{Cl} 6$ & 35 & $\mathrm{CIO} 2$ \\
\hline 14 & $\mathrm{C} 4 / \mathrm{C} 2$ & 36 & $\mathrm{Cl} 4: 2$ \\
\hline 15 & C4/C3 & 37 & CI8:2 \\
\hline 16 & $\mathrm{C} 4 / \mathrm{C} 8$ & 38 & $\mathrm{Cl0} 2 / \mathrm{ClO}$ \\
\hline 17 & $\mathrm{C} 5 / \mathrm{CO}$ & 39 & C6DC \\
\hline 18 & $\mathrm{C} 5 / \mathrm{C} 2$ & 40 & C5DC/C8 \\
\hline 19 & $\mathrm{C} 5 / \mathrm{C} 3$ & 41 & $(0+2+3+16+18: 1) / C i t$ \\
\hline 20 & $\mathrm{C} 5-\mathrm{OH} / \mathrm{C} 8$ & 42 & $(\mathrm{Cl} 6+\mathrm{Cl}) / \mathrm{C} 0$ \\
\hline 21 & $\mathrm{C} 5-\mathrm{OH} / \mathrm{CO}$ & 43 & $\mathrm{CO} /(\mathrm{Cl} 6+\mathrm{Cl})$ \\
\hline 22 & $\mathrm{C} 5 \mathrm{DC} / \mathrm{C} 5-\mathrm{OH}$ & 44 & C3/Met \\
\hline
\end{tabular}

\section{Publish your work in this journal}

OncoTargets and Therapy is an international, peer-reviewed, open access journal focusing on the pathological basis of all cancers, potential targets for therapy and treatment protocols employed to improve the management of cancer patients. The journal also focuses on the impact of management programs and new therapeutic agents and protocols on patient perspectives such as quality of life, adherence and satisfaction. The manuscript management system is completely online and includes a very quick and fair peer-review system, which is all easy to use. Visit http://www.dovepress.com/testimonials.php to read real quotes from published authors. 